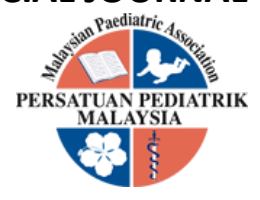

\title{
CHRONIC GRANULOMATOUS DISEASE
}

\author{
Zarina Thasneem Zainudeen, Ilie Fadzilah Hashim, Intan Juliana Abd Hamid
}

Received: 6 December 2019; Accepted revised manuscript: 20 January 2020

Published online: 4 February 2020

\begin{abstract}
Chronic granulomatous disease (CGD) is defined as an inherited phagocyte disorder causing defective superoxide generation and intracellular killing. Reduced or missing burst activity of nicotinamide dinucleotide phosphate (NADPH) oxidase complex is observed in this inborn defect that usually manifests itself during the first two years of life. It can be inherited either by X-linked inheritance or autosomal recessive inheritance. Most patients with CGD develop failure to thrive, severe bacterial adenitis, abscesses, osteomyelitis or hyperinflammatory manifestations.
\end{abstract}

PHAGOBURSTTM allows the quantitative determination of leukocyte oxidative burst. This kit contains unlabeled opsonized E. coli bacteria as particulate stimulus, the protein kinase $C$ ligand phorbol 12-myristate 13 acetate (PMA) as high stimulus and the chemotactic peptide $\mathrm{N}$-formyl-MetLeuPhe (fMLP) as low physiological stimulus and dihydrorhodamine (DHR )123 as a fluorogenic substrate and necessary reagents.

Heparinized whole blood is incubated with the various stimuli at $370 \mathrm{C}$, a sample without stimulus serves as negative background control. Upon stimulation, granulocyte and monocytes produce reactive oxygen metabolites (superoxide anion, hydrogen peroxide, hypochlorous acid) which destroy bacteria inside phagosome. Formation of the reactive oxidants during the oxidative burst can be monitored by the addition and oxidation of DHR 123. The reaction is stopped by addition of lysing solution, which removes erythrocytes and results in a partial fixation of leukocytes. After a washing step with wash solution, DNA staining solution is added to exclude aggregation artifacts of bacteria or cell. The percentage of cells having produced reactive oxygen radicals are then analyzed as well as their mean fluorescence intensity (enzymatic activity).

The oxidation of dihydrorhodamine (DHR) to fluorescent rhodamine is measured by FACS Canto II at AMDI, USM. The unstimulated fluorescence is represented by the red histogram, while the PMA-stimulated oxidative burst in neutrophils is represented by blue histogram. (A) Granulocytes are gated based on their inherent characteristic light scatter properties by forward/side scatter. (B)

Primary Immunodeficiency Diseases Group, Cluster of Regenerative Medicine, Advanced Medical and Dental Institute (AMDI), Universiti Sains Malaysia, Penang.

Corresponding author: Zarina Thasneem Zainudeen,

Advanced Medical and Dental Institute (AMDI),

SAINS@Bertam, Universiti Sains Malaysia, 13200, Kepala

Batas, Penang, Malaysia.

Email: zarina.zainudeen@usm.my; Tel: +604-5622175 

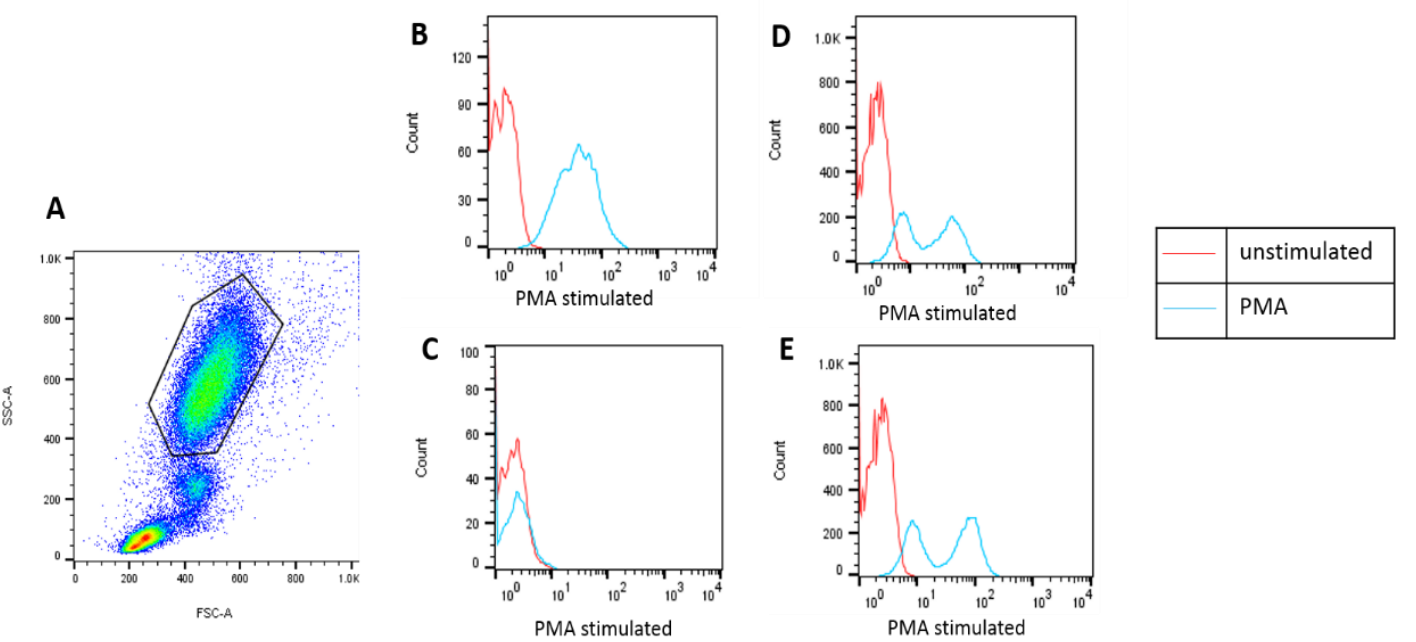

Figure 1: Results of our flow cytometry-based oxidative burst assay for the diagnosis of X-linked CGD in a family.

DHR flow cytometry assay histograms demonstrating the change in fluorescence in neutrophils following PMA stimulation in a control subject. (C) Absence of change in fluorescence in neutrophils following PMA stimulation in a patient with X-linked recessive CGD. (D) a X-linked carrier mother. (E) a X-linked carrier sister. Both mother and sister clearly showed two peaks, one normal and one abnormal. The results on the mother clarified the probable patient diagnosis and were reported as consistent with $\mathrm{X}$-linked CGD for the son.

\section{Conclusion}

The DHR flow assay is useful in evaluating the oxidative burst activity of granulocytes from CGD patients defective in NADPH oxidase function. This assay offers cheap and rapid alternative of diagnosing $X$-linked CGD cases. This assay also useful in evaluating for carrier status in female relatives of patients with $X$ linked CGD. Initial results can help the physician determine what other investigative procedures are needed to arrive at a definite diagnosis and initiate the necessary actions such as deferring the live vaccines administration, starting antibiotic prophylaxis, early donor search and preparation for allogeneic hematopoietic stem cell transplantation.

\section{Disclaimer}

This assay was done using PhagoburstTM kit and analyzed on BD FACS Canto $\|$ at Advanced Medical and Dental Institute, USM, Penang. 